\title{
Virulence Variations of Puccinia striiformis f. sp. tritici Isolates Collected from Berberis spp. in China
}

Zhiyan Wang and Jie Zhao, State Key Laboratory of Crop Stress Biology for Arid Areas and College of Plant Protection, Northwest A\&F University, Yangling, Shaanxi 712100, P. R. China; Xianming Chen, United States Department of Agriculture-Agricultural Research Service, Wheat Genetics, Quality, Physiology, and Disease Research Unit and Department of Plant Pathology, Washington State University, Pullman 99164-6430; Yuelin Peng, Department of Plant Sciences, Agricultural and Animal Husbandry College of Tibet University, Linzhi, Tibet 86000, P. R. China; and Jingjing Ji, Shilei Zhao, Yanjie Lv, Lili Huang, and Zhensheng Kang, State Key Laboratory of Crop Stress Biology for Arid Areas and College of Plant Protection, Northwest A\&F University

\begin{abstract}
Wang, Z. Y., Zhao, J., Chen, X. M., Peng, Y. L., Ji, J. J., Zhao, S. L., Lv, Y. J., Huang, L. L., and Kang, Z. S. 2016. Virulence variations of Puccinia striiformis f. sp. tritici isolates collected from Berberis spp. in China. Plant Dis. 100:131-138.

The stripe rust pathogen Puccinia striiformis f. sp. tritici frequently causes significant yield losses in China, due to rapid development of new races that overcome resistance in wheat cultivars. Indirect evidence suggests that sexual reproduction occurs in the $P$. striiformis $\mathrm{f}$. sp. tritici population in China but direct evidence was still lacking. In this study, a large-scale survey of barberry (Berberis spp.) was conducted in Gansu, Shaanxi, Tibet, and Xinjiang provinces in western China. In total, 9,297 single-aecial (SA) samples were used to inoculate a susceptible wheat cultivar to identify samples of $P$. striiformis f. sp. tritici. Sixteen of the SA samples were identified as $P$. striiformis $\mathrm{f}$. sp. tritici. When tested on the wheat differentials for identifying $P$. striiformis f. sp. tritici races, 15 of the 16 SA samples had different virulence patterns, indicating that they were sexually produced through barberry. From the 16 SA samples, 118 single-uredinium (SU)

isolates were obtained, from which 88 virulence patterns were identified when tested on $17 \mathrm{Yr}$ single-gene lines. The virulence patterns had relatively narrow virulence spectra, ranging from 0 to 9 , with a mean of four virulences per SU isolates. Of the $17 \mathrm{Yr}$ genes, no virulences were detected for $Y r 5, \operatorname{Yr} 10$, and $Y r 15$; virulences to $Y r T r 1, Y r 24$, and $Y r 27$ were extremely low $(<3 \%)$; those to $Y r S P, Y r 9, Y r 28$, and $Y r 2$ were low (13.6 to 28.0\%); those to $Y r 7, Y r 17, Y r 8$, and $Y r E x p 2$ were moderate (33.1 to 48.3\%); and those to $Y r 6, Y r 44$, and $Y r 25$ were high (52.5 to $72.9 \%)$. This study provides direct evidence that natural sexual reproduction occurs in the P. striiformis $\mathrm{f}$. sp. tritici population in China, but the frequency appears to be very low. The sexual reproduction on alternate host plants can generate a great virulence diversity, which may have contributed to the high variation in the P. striiformis f. sp. tritici population in China.
\end{abstract}

Stripe rust (yellow rust), caused by the Basidiomycete fungus Puccinia striiformis f. sp. tritici Erikss., is one of the most damaging wheat diseases in the world (Chen 2005; Kang et al. 2010; Rapilly 1979; Wellings 2011). In China, $P$. striiformis f. sp. tritici has caused several large-scale epidemics resulting in huge yield losses (Li and Zeng 2002). Although fungicides are being used to reduce yield losses, developing resistant cultivars is considered to be the most effective approach for control of the disease (Chen et al. 2014; Wan et al. 2004). However, numerous $P$. striiformis f. sp. tritici races have been identified, which have overcome resistance in many wheat cultivars that are grown widely (Chen et al. 2009; Hu et al. 2012; Kang et al. 1994, 2010; Li and Zeng 2002; Wan et al. 2003, 2004). It is still a challenge to develop wheat cultivars with long-lasting resistance because new races appear rapidly (Chen et al. 2009; Hu et al. 2012; Kang et al. 2010).

New races of $P$. striiformis f. sp. tritici are generated through mutation and somatic recombination, and also possibly through sexual recombination. The stripe rust pathogen accumulates a large number of virulences in single isolates or races through both mutation and clonal production (Chen 2005; Chen et al. 2009; Kang et al. 1994; Line and Qayoum 1992; Wellings and McIntosh 1990). In addition, introduction (natural or human mediated) of new P. striiformis f. sp. tritici races is realized by interregional dissemination through airborne

Corresponding authors: Z. S. Kang; E-mail: kangzs@nwsuaf.edu.cn; and L. L. Huang; E-mail: huanglili@nwsuaf.edu.cn

Z. Wang and J. Zhao contributed equally to this work.

Accepted for publication 12 July 2015.

http://dx.doi.org/10.1094/PDIS-12-14-1296-RE

(C) 2016 The American Phytopathological Society spores or human activities (Chen 2005; Wellings 2011). Based on the heterozygosis of $P$. striiformis $\mathrm{f}$. sp. tritici, isolates of different races are revealed to combine their virulences to form new races with broad virulence spectra through somatic recombination during the germination and infection processes on or in host tissue (Harder 1984; Kang et al. 1993; Little and Manners 1969; Rodenhiser and Hard-Karrer 1947). A series of previous studies uncovered that the Chinese $P$. striiformis f. sp. tritici population has a much higher genotypic and virulence diversity than other clonal $P$. striiformis f. sp. tritici populations (Duan et al. 2010; Lu et al. 2009; Mboup et al. 2009; Shan et al. 1998; Zheng et al. 2013), suggesting sexual reproduction involving the Chinese $P$. striiformis $\mathrm{f}$. sp. tritici population but lacking direct evidence from alternate hosts. Recently, the sexual recombination of $P$. striiformis $\mathrm{f}$. sp. tritici was indicated based on the high genetic diversity and recombinant population structure in the Himalayan region of Pakistan, adjacent to the western boundary of China (Ali et al. 2014). Virulence variations are determined not only by the composition of races but also the frequency changes of individual races. Frequency changes can be caused by host selection and genetic drift, and also result from differences of races in fitness and aggressiveness (Chen et al. 2010; Milus et al. 2006; Wan and Chen 2014).

The discovery of a sexual host for the stripe rust fungus by Jin et al. (2010) demonstrated that the $P$. striiformis species complex has a sexual cycle. Prior to the discovery, the sexual stage of this rust fungus was thought to be absent (Rapilly 1979; Stubbs 1985). Subsequently, more Berberis spp. (Zhao et al. 2013) and Mahonia spp. (Wang and Chen 2013) were identified as alternate hosts for $P$. striiformis f. sp. tritici by means of artificial inoculation.

In China, approximately 250 Berberis spp. have been described (Ying and Chen 2001). Based on our investigations, abundant barberry plants grow wild along mountain slopes and around fields in northwestern and southwestern China (Zhao et al. 2013). Abundant aecia are often found on barberry in these regions and stripe rust is 
the most common rust disease of wheat; thus, it is likely that $P$. striiformis f. sp. tritici is cycling through the sexual cycle on barberry. Because the genetic diversity of the $P$. striiformis $\mathrm{f}$. sp. tritici population in China is high (Kang et al. 1987; Lu et al. 2009; Mboup et al. 2009; Shan et al. 1998; Sharma-Poudyal et al. 2013; Zhan et al. 2012; Zheng et al. $2005,2013)$, the fungus is likely to have sexual reproduction. Under controlled conditions, Zhao et al. (2013) demonstrated that $P$. striiformis f. sp. tritici is able to infect a large number of Berberis spp. They obtained four $P$. striiformis f. sp. tritici aecial isolates from naturally infected barberry plants in Gansu and Shaanxi provinces. By inoculating the aecial isolates on wheat plants, they identified a large number of races. However, none of them were the same as races that have been identified from wheat fields. The number of aecial isolates was too small to determine how important barberry is for wheat stripe rust epidemics. Thus, a large-scale survey is needed to determine how intensively barberry is infected by the stripe rust fungus under natural conditions in western China.

The specific objectives of this study were to (i) isolate $P$. striiformis f. sp. tritici samples from naturally infected barberry plants and (ii) identify virulence characteristics of the $P$. striiformis f. sp. tritici samples and their single-uredinium (SU) isolates on differentials to determine pathotype and diversity in pathogenicity. The results should provide more insight as to the role of alternate hosts in wheat stripe rust epidemics and generation of diverse races.

\section{Materials and Methods}

Aecial samples. Surveys of rust infection on barberry plants were conducted in Gansu, Shaanxi, Tibet, and Xinjiang provinces in northwestern and southwestern China from April to June 2013. Barberry leaves bearing mature and fresh aecia were collected from bushes of infected Berberis spp. at each of the sampling sites in these provinces, placed in paper bags, and kept in a cool box. After being brought back to the laboratory, the samples were either immediately used to inoculate wheat plants or kept in sealed plastic bags containing desiccant at $4^{\circ} \mathrm{C}$ for later use.

Inoculation of wheat plants with aeciospores. Wheat 'Mingxian 169', susceptible to all Chinese $P$. striiformis f. sp. tritici races, was grown in plastic pots $(7$ by 7 by $8 \mathrm{~cm}$ ) in a rust-free growth chamber set at a temperature of $16 \pm 3^{\circ} \mathrm{C}$ and a photoperiod of $16 \mathrm{~h}$ of light and $8 \mathrm{~h}$ of darkness. A single aecium (SA) was cut from a barberry leaf using a blade, placed onto a glass slide with two to three drops of deionized water, and crushed gently using an inoculation needle to release aeciospores, forming aeciospore suspensions that were inoculated onto the adaxial surface of primary wheat leaves using a swab. To minimize contamination, the inoculated plants were covered using a transparent plastic cylinder with an open top, and kept for 24 to $36 \mathrm{~h}$ at $10^{\circ} \mathrm{C}$ in darkness in a dew chamber. After incubation, the plants were transferred to a clean growth chamber set to a diurnal cycle of $16^{\circ} \mathrm{C}$ for the 16 -h light period and $13^{\circ} \mathrm{C}$ for the 8 -h dark time. Symptoms and signs were observed and infection types (IT) were recorded based on the 0-to- 9 scale for $P$. striiformis f. sp. tritici (Line and Qayoum 1992) and the 0-to-4 scale for $P$. graminis f. sp. tritici (Stakman et al. 1962) 15 to 20 days after inoculation. Urediniospores were collected from wheat leaves inoculated with aeciospores from each aecium and treated as an SA sample.

Establishment of SU population. The wheat leaves inoculated with aeciospores were kept for 5 to 8 days for collecting urediniospores to represent the SA samples. For each SA sample, 10 to 14 fresh uredinia were randomly picked from wheat leaves inoculated with aeciospores using a metal office pin tipped with a small amount of Vaseline jelly to inoculate new wheat leaves for establishing an SU isolate population. To ensure that urediniospores were from an SU, uredinia that were not completely erupted through the host tissue were used. The resultant urediniospores were used to inoculate new Mingxian 169 seedlings with a fine brush with deionized water for increasing spores. The inoculated wheat plants were kept in a dew chamber at $10^{\circ} \mathrm{C}$ for $24 \mathrm{~h}$, and grown in a growth chamber at 13 to $16^{\circ} \mathrm{C}$, with a photoperiod of $16 \mathrm{~h}$ of light and $8 \mathrm{~h}$ of darkness. If the amount of urediniospores was not enough, urediniospore multiplication was repeated following the method mentioned above.

Determination of virulence phenotypes of $\boldsymbol{P}$. striiformis $\mathbf{f}$. $\mathbf{s p}$. tritici isolates. The virulence patterns of the SA samples were determined by inoculating fresh urediniospores onto seedlings of the set of 19 wheat genotypes used to differentiate Chinese P. striiformis f. sp. tritici races, according to the method described by Chen et al. (2009). Similarly, the virulence patterns of the SA and SU isolates were determined on $17 \mathrm{Yr}$ single-gene lines, most of which have been

Table 2. Identification of Puccinia striiformis f. sp. tritici and P. graminis f. sp. tritici aecial samples recovered by inoculation of Mingxian 169 wheat with aeciospores of single aecium collected from naturally infected barberry plants in 2013

\begin{tabular}{|c|c|c|}
\hline Samples & $\begin{array}{c}\text { Number of } \\
\text { aecial samples }\end{array}$ & Percentage $(\%)$ \\
\hline Puccinia striiformis f. sp. tritici (IT 7-9) ${ }^{\mathrm{a}}$ & 16 & 0.17 \\
\hline P. graminis f. sp. tritici $\left(\right.$ IT 3-4) ${ }^{\mathrm{b}}$ & 12 & 0.13 \\
\hline Unknown & 9,269 & 99.70 \\
\hline Symptomless (IT 0) & 9,078 & 97.65 \\
\hline Necrotic spots (IT 0;) & 191 & 2.05 \\
\hline Total & 9,297 & 100.00 \\
\hline
\end{tabular}

a Infection types (IT) 7 to 9, based on a 0-to-9 scale (Line and Qayoum 1992), are considered virulent.

b IT 3 to 4, based on a 0-to-4 scale (Stakman et al. 1962), are considered virulent.

Table 1. Number of samples of Puccinia striiformis f. sp. tritici and P. graminis f. sp. tritici recovered from aecia on Berberis spp. collected from Gansu, Shaanxi, Tibet, and Xinjiang provinces in 2013

\begin{tabular}{llcccc}
\hline & & & & \multicolumn{2}{c}{ Number of samples/sites } \\
\cline { 3 - 6 } Berberis spp. & Origin & Berberis spp. (bushes/sites) & Number of aecia & P. striiformis f. sp. tritici & P. graminis f. sp. tritici \\
\hline Berberis aggregata & Gansu & $43 / 5$ & 3,059 & $8 / 3^{\text {b }}$ & $5 / 2$ \\
B. brachypoda & Gansu & $13 / 4$ & 1,763 & 0 & $4 / 2$ \\
B. heteropoda & Xinjiang & $11 / 4$ & 102 & 0 & 0 \\
B. polyantha & Tibet & $26 / 6$ & 2,228 & $4 / 3^{c}$ & $3 / 2$ \\
B. shensiana & Shaanxi & $45 / 5$ & 1,832 & 0 & $2 / 2$ \\
B. soulieana & Gansu & $13 / 2$ & 281 & $16 / 9$ & 0 \\
Total & $\ldots$ & $151 / 26$ & 9,297 & $14 / 8$ \\
\hline
\end{tabular}

${ }^{a}$ Number of bushes and sites of Berberis spp. samples. The distance among sites where aecial samples were collected is approximate 5 to $40 \mathrm{~km}$.

b The three sites from which the eight $P$. striiformis f. sp. tritici aecial samples were collected from B. aggregata were at a hillside and a hill foot near the Lvjiapo village and near the Liuluo village of Tianshui in Gansu Province.

${ }^{c}$ The three sites where the four P. striiformis f. sp. tritici aecial samples were collected from B. polyantha were in Milin County, near the Bujiu village of Linzhi County, and the Mirui village of Linzhi County in Tibet.

$\mathrm{d}$ The three sites where the four aecial samples were collected from B. shensiana, were from the two different locations near the Guan village and the Caiyuan village of Baoji County in Shaanxi Province. 
used to differentiate races in the United States and other countries (Sharma-Poudyal et al. 2013; Wan and Chen 2014). The procedures and conditions for inoculation and growing inoculated plants were the same as described above. The IT data were recorded using the 0 -to-9 scale for $P$. striiformis f. sp. tritici and 0-to-4 scale for $P$. graminis f. sp. tritici as described above.

\section{Results}

SA samples of $\boldsymbol{P}$. striiformis f. sp. tritici from barberry plants. Six Berberis spp. were surveyed at 26 sites located in four provinces in this study. In total, 9,297 single aecia were collected from 151 bushes and tested on susceptible Mingxian 169 wheat (Table 1). Of the collected aecial samples, 16 aecia produced typical $P$. striiformis f. sp. tritici uredinia with high IT, resulting in 16 SA samples, designated as U1 to U16. The 16 SA samples were obtained from three Berberis spp.: Berberis aggregata in Gansu, B. polyantha in Tibet, and $B$. shensiana in Shaanxi. No $P$. striiformis $\mathrm{f}$. sp. tritici isolates were obtained from aecial samples from Xinjiang. This indicated that $P$. striiformis $\mathrm{f}$. sp. tritici was collected at a low overall frequency of $0.17 \%$. Most of the aecial samples inoculated on Mingxian 169 produced necrotic flecks without uredinia (IT 1 to 2 using the 0-to-9 scale for $P$. striiformis f. sp. tritici or IT 0; using the

Table 3. Avirulence and virulence patterns of 16 Puccinia striiformis $\mathrm{f}$. sp. tritici samples obtained from 16 single aecium produced on naturally infected Berberis spp. on the set of 19 wheat cultivars used to differentiated $P$. striiformis f. sp. tritici races

\begin{tabular}{|c|c|c|c|c|c|c|c|c|c|c|c|c|c|c|c|c|c|c|c|c|}
\hline \multirow[b]{2}{*}{ Sample } & \multirow[b]{2}{*}{ Berberis spp. } & \multicolumn{19}{|c|}{ Avirulence and virulence on differentials ${ }^{\mathbf{a}}$} \\
\hline & & 1 & 2 & 3 & 4 & 5 & 6 & 7 & 8 & 9 & 10 & 11 & 12 & 13 & 14 & 15 & 16 & 17 & 18 & $\overline{19}$ \\
\hline $\mathrm{U} 1$ & B. aggregata & A & $\mathrm{V}$ & $\mathrm{V}$ & $\mathrm{V}$ & $\mathrm{V}$ & $\mathrm{V}$ & $\mathrm{V}$ & $\mathrm{V}$ & A & $\mathrm{V}$ & $\mathrm{AV}$ & A & A & $\mathrm{V}$ & A & A & A & A & A \\
\hline $\mathrm{U} 2$ & B. aggregata & $\mathrm{V}$ & $\mathrm{V}$ & $\mathrm{V}$ & V & $\mathrm{V}$ & V & V & $\mathrm{V}$ & V & $\mathrm{V}$ & $\mathrm{V}$ & $\mathrm{V}$ & $\mathrm{V}$ & V & A & $\mathrm{V}$ & V & A & A \\
\hline $\mathrm{U} 3$ & B. aggregata & V & V & V & V & V & V & V & V & V & V & $\mathrm{V}$ & $\mathrm{V}$ & V & V & A & $\mathrm{V}$ & V & A & A \\
\hline U4 & B. polyantha & A & V & A & V & A & V & V & V & A & A & AV & A & A & V & A & A & A & A & A \\
\hline U5 & B. shensiana & A & V & A & V & V & V & $\mathrm{V}$ & V & A & V & A & A & A & V & A & A & A & A & A \\
\hline U6 & B. aggregata & VA & $\mathrm{A}$ & $\mathrm{V}$ & V & AV & V & V & V & A & $\mathrm{V}$ & $\mathrm{V}$ & $\mathrm{V}$ & A & $\mathrm{V}$ & A & $\mathrm{V}$ & A & A & A \\
\hline U7 & B. shensiana & A & $\mathrm{V}$ & A & V & $\mathrm{V}$ & V & V & $\mathrm{V}$ & A & $\mathrm{AV}$ & $\mathrm{AV}$ & A & A & $\mathrm{V}$ & A & A & A & A & A \\
\hline U8 & B. polyantha & A & $\mathrm{A}$ & $\mathrm{A}$ & V & A & V & $\mathrm{AV}$ & $\mathrm{AV}$ & A & $\mathrm{V}$ & $\mathrm{V}$ & A & A & $\mathrm{V}$ & $\mathrm{A}$ & A & A & A & A \\
\hline U9 & B. shensiana & $\mathrm{V}$ & A & V & $\mathrm{AV}$ & A & V & $\mathrm{V}$ & VA & A & $\mathrm{V}$ & $\mathrm{AV}$ & $\mathrm{V}$ & V & A & $\mathrm{A}$ & A & A & A & A \\
\hline U10 & B. shensiana & $\mathrm{V}$ & $\mathrm{V}$ & $\mathrm{V}$ & V & $\mathrm{V}$ & V & $\mathrm{V}$ & $\mathrm{V}$ & A & $\mathrm{V}$ & $\mathrm{V}$ & $\mathrm{V}$ & $\mathrm{V}$ & $\mathrm{V}$ & A & $\mathrm{V}$ & $\mathrm{V}$ & A & A \\
\hline U11 & B. aggregata & A & A & A & V & A & V & $\mathrm{AV}$ & A & A & $\mathrm{V}$ & $\mathrm{V}$ & A & A & VA & $\mathrm{A}$ & A & $\mathrm{A}$ & A & A \\
\hline U12 & B. polyantha & $\mathrm{A}$ & A & A & $\mathrm{AV}$ & A & V & VA & A & A & $\mathrm{V}$ & $\mathrm{AV}$ & A & A & $\mathrm{V}$ & $\mathrm{A}$ & A & A & A & A \\
\hline U13 & B. polyantha & $\mathrm{A}$ & $\mathrm{A}$ & $\mathrm{AV}$ & $\mathrm{AV}$ & A & $\mathrm{AV}$ & $\mathrm{AV}$ & A & $\mathrm{A}$ & $\mathrm{V}$ & A & A & AV & VA & A & A & $\mathrm{A}$ & A & A \\
\hline U14 & B. aggregata & V & V & V & $\mathrm{V}$ & $\mathrm{V}$ & $\mathrm{V}$ & $\mathrm{V}$ & V & A & $\mathrm{V}$ & $\mathrm{V}$ & $\mathrm{AV}$ & A & $\mathrm{V}$ & A & A & A & A & A \\
\hline U15 & B. aggregata & $\mathrm{A}$ & A & A & $\mathrm{AV}$ & A & A & V & A & A & $\mathrm{V}$ & A & A & A & V & $\mathrm{A}$ & A & A & A & A \\
\hline U16 & B. aggregata & A & $\mathrm{A}$ & $\mathrm{AV}$ & $\mathrm{AV}$ & A & V & VA & A & A & $\mathrm{V}$ & $\mathrm{AV}$ & A & A & V & A & $\mathrm{A}$ & A & A & A \\
\hline
\end{tabular}

${ }^{a}$ Wheat differentials: $1=$ Trigo Eureka, $2=$ Fulhard, $3=$ Lutescenes 128, $4=$ Mentana, $5=$ Virgilio, $6=$ Abbondanza, $7=$ Early Piemium, $8=$ Funo, $9=$ Danish 1 , $10=$ Jubilejina 2, $11=$ Fengchan 3, $12=$ Lovrin 13, $13=$ Kangyin $655,14=$ Suwon 11, $15=$ Zhong 4, $16=$ Lovrin 10, $17=$ Hybrid 46, $18=$ T. spelta ablum, $19=$ Guinong 22. $A=$ avirulent (infection types 0 to 6); $V=$ virulence (infection types 7-9); and $A V$ or VA = mixed infection types, with more avirulent reactions in $\mathrm{AV}$ and more virulent reaction in VA.

${ }^{\text {b }}$ Samples U1 to U16 are P. striiformis f. sp. tritici obtained from Mingxian 169 wheat inoculated with aeciospores of single-aecium populations.

Table 4. Avirulence and virulence patterns of 16 Puccinia striiformis f. sp. tritici samples obtained from 16 single aecium on wheat $Y r$ single gene lines

\begin{tabular}{|c|c|c|c|c|c|c|c|c|c|c|c|c|c|c|c|c|c|c|c|}
\hline \multirow[b]{2}{*}{ Sample $^{\mathbf{c}}$} & \multirow[b]{2}{*}{ Yr2 } & \multicolumn{16}{|c|}{ Avirulence or virulence on wheat $Y r$ single-gene lines ${ }^{a}$} & \multicolumn{2}{|c|}{$\begin{array}{c}\text { Number } \\
\text { of }^{\mathbf{b}}\end{array}$} \\
\hline & & Yr5 & Yr6 & Yr7 & Yr8 & Yr9 & Yr10 & Yr15 & Yr17 & Yr24 & Yr27 & Yr44 & YrSP & YrTr1 & YrExp2 & Yr25 & Yr28 & SU & VP \\
\hline U1 & A & A & AV & $\mathrm{AV}$ & VA & A & A & A & $\mathrm{AV}$ & A & A & $\mathrm{AV}$ & A & A & VA & $\mathrm{AV}$ & $\mathrm{AV}$ & 14 & 12 \\
\hline U2 & $\mathrm{AV}$ & A & AV & $\mathrm{AV}$ & AV & $\mathrm{AV}$ & A & A & AV & $\mathrm{AV}$ & A & $\mathrm{AV}$ & AV & A & AV & VA & $\mathrm{AV}$ & 13 & 12 \\
\hline U3 & VA & A & $\mathrm{V}$ & A & A & $\mathrm{V}$ & A & A & V & A & $\mathrm{AV}$ & $\mathrm{V}$ & $\mathrm{V}$ & A & A & V & VA & 5 & 3 \\
\hline U4 & A & A & $\mathrm{AV}$ & VA & $\mathrm{V}$ & A & A & A & A & A & A & VA & A & A & VA & $\mathrm{V}$ & A & 3 & 3 \\
\hline U5 & AV & A & $\mathrm{AV}$ & $\mathrm{AV}$ & $\mathrm{AV}$ & A & A & A & AV & A & A & VA & A & A & VA & VA & $\mathrm{AV}$ & 9 & 9 \\
\hline U6 & VA & A & VA & A & A & VA & A & A & AV & A & $\mathrm{AV}$ & VA & $\mathrm{AV}$ & A & A & VA & VA & 5 & 5 \\
\hline U7 & $\mathrm{V}$ & A & VA & VA & VA & A & A & A & A & A & A & VA & A & A & $\mathrm{V}$ & $\mathrm{V}$ & A & 5 & 4 \\
\hline U8 & $\mathrm{AV}$ & A & VA & VA & $\mathrm{V}$ & A & A & A & $\mathrm{AV}$ & A & A & VA & A & A & $\mathrm{V}$ & $\mathrm{V}$ & A & 5 & 5 \\
\hline U9 & $\mathrm{AV}$ & A & VA & A & A & VA & A & A & $\mathrm{AV}$ & A & $\mathrm{AV}$ & VA & $\mathrm{AV}$ & A & A & VA & VA & 9 & 9 \\
\hline U10 & $\mathrm{AV}$ & A & VA & A & A & VA & A & A & VA & A & A & VA & VA & A & A & $\mathrm{AV}$ & A & 2 & 2 \\
\hline U11 & $\mathrm{AV}$ & A & VA & VA & VA & $\mathrm{AV}$ & A & A & AV & $\mathrm{AV}$ & A & VA & $\mathrm{A}$ & A & VA & VA & $\mathrm{AV}$ & 10 & 10 \\
\hline U12 & A & A & VA & VA & VA & $\mathrm{AV}$ & A & A & $\mathrm{AV}$ & A & A & VA & AV & A & VA & VA & $\mathrm{A}$ & 6 & 6 \\
\hline U13 & AV & A & $\mathrm{AV}$ & VA & VA & $\mathrm{AV}$ & A & A & AV & A & A & AV & $\mathrm{AV}$ & A & VA & VA & $\mathrm{AV}$ & 11 & 10 \\
\hline U14 & $\mathrm{AV}$ & A & VA & $\mathrm{AV}$ & VA & A & A & A & VA & A & A & VA & A & A & VA & VA & A & 6 & 6 \\
\hline U15 & $\mathrm{AV}$ & A & $\mathrm{AV}$ & $\mathrm{AV}$ & VA & $\mathrm{AV}$ & A & A & VA & $\mathrm{AV}$ & A & VA & A & A & VA & VA & A & 11 & 10 \\
\hline U16 & VA & A & VA & VA & $\mathrm{AV}$ & $\mathrm{AV}$ & A & A & $\mathrm{AV}$ & A & A & $\mathrm{V}$ & A & A & AV & $\mathrm{V}$ & A & 4 & 4 \\
\hline Total & & & & & & & & & & & & & & & & & & 118 & 88 \\
\hline
\end{tabular}

a Wheat $Y r$ single-gene lines: $Y r 2$, Kalyansona; $Y r 5$, AvSYr5NIL; Yr6, AvSYr6NIL; Yr7, AvSYr7NIL; $Y r 8$, AvSYr8NIL; $Y r 9$, AvSYr9NIL; Yr10, AvSYr10NIL; Yr15, AvSYr15NIL; Yr17, AvSYr17NIL, Yr24, AvSYr24NIL; Yr27, AvSYr27NIL; Yr44, AvS/Zak (1-1-35-line1); YrSP, AvSYrSPNIL; YrTr1, AvSYrTr1NIL; YrExp2, AvS/Exp 1/1-1 Line 74; Yr25, Hugenoot; Yr28, AvSYr28NIL (Sharma-Poudyal et al. 2013; Wan and Chen 2014). A = avirulent (infection types 0 to 6 ), $\mathrm{V}=$ virulent (infection types 7 to 9 ), $\mathrm{AV}=$ more avirulent infection types than virulent infection types on the same leaves, and $\mathrm{VA}=$ more virulent infection types than avirulent infection types.

${ }^{\mathrm{b}} \mathrm{SU}=$ single uredinium isolates tested and $\mathrm{VP}=$ virulence patterns identified.

c Samples U1 to U16 are $P$. striiformis f. sp. tritici derived from 16 single aecium collected from naturally infected barberry plants. 
Table 5. Avirulence and virulence patterns of 118 single uredinial isolates of Puccinia striiformis f. sp. tritici obtained from 16 single-aecium samples collected from naturally infected Berberis spp.

Avirulence (A) or virulence (V) on $\mathrm{Yr}$ single-gene lines

\begin{tabular}{|c|c|c|c|c|c|c|c|c|c|c|c|c|c|c|c|c|c|c|}
\hline Isolate $^{b}$ & $Y r 2$ & Yr5 & Yr6 & $Y r 7$ & Yr8 & $\operatorname{Yr} 9$ & Yr10 & Yr15 & Yr17 & Yr24 & Yr27 & Yr44 & YrSP & YrTr1 & YrExp2 & Yr25 & Yr28 & $V P^{c}$ \\
\hline U1-1 & A & A & A & A & $\mathrm{V}$ & A & A & A & A & A & A & A & A & A & $\mathrm{V}$ & A & A & VP-1 \\
\hline U1-2 & A & A & A & A & $\mathrm{V}$ & A & A & A & A & A & A & $\mathrm{V}$ & A & A & A & $\mathrm{V}$ & A & VP-2 \\
\hline U1-3 & A & A & $\mathrm{V}$ & A & $\mathrm{V}$ & A & A & A & A & A & A & A & A & A & A & A & A & VP-3 \\
\hline U1-4 & A & A & A & A & A & A & A & A & $\mathrm{V}$ & A & A & V & A & A & $\mathrm{V}$ & $\mathrm{V}$ & A & VP-4 \\
\hline U1-5 & A & A & A & A & $\mathrm{V}$ & A & A & A & A & A & A & A & A & A & A & A & $\mathrm{V}$ & VP-5 \\
\hline U1-6 & A & A & V & $\mathrm{V}$ & $\mathrm{V}$ & A & A & A & A & A & A & $\mathrm{V}$ & A & A & $\mathrm{V}$ & $\mathrm{V}$ & A & VP-6 \\
\hline U1-7 & A & A & A & A & A & A & A & A & A & A & A & A & A & A & A & A & A & VP-7 \\
\hline U1-8 & $\mathrm{V}$ & A & V & A & $\mathrm{V}$ & A & A & A & V & A & A & V & A & A & $\mathrm{V}$ & $\mathrm{V}$ & A & VP-8 \\
\hline U1-9 & A & A & A & A & $\mathrm{V}$ & A & A & A & A & A & A & $\mathrm{V}$ & A & A & $\mathrm{V}$ & A & A & VP-9 \\
\hline U1-10 & A & A & A & A & A & A & A & A & A & A & A & A & A & A & A & A & A & VP-7 \\
\hline U1-11 & A & A & A & $\mathrm{V}$ & $\mathrm{V}$ & A & A & A & A & A & A & A & A & A & A & A & A & VP-10 \\
\hline U1-12 & A & A & A & A & V & A & A & A & A & A & A & A & A & A & $\mathrm{V}$ & A & A & VP1 \\
\hline U1-13 & A & A & A & A & $\mathrm{V}$ & A & A & A & V & A & A & A & A & A & V & $\mathrm{V}$ & A & VP-11 \\
\hline U1-14 & A & $\mathrm{A}$ & A & A & $\mathrm{V}$ & A & A & A & A & A & A & A & A & A & V & A & $\mathrm{V}$ & VP-12 \\
\hline U2-1 & A & $\mathrm{A}$ & A & A & A & A & A & A & A & A & A & A & A & A & A & A & A & VP-7 \\
\hline $\mathrm{U} 2-2$ & A & $\mathrm{A}$ & V & V & V & A & A & A & A & A & A & A & A & A & V & V & A & VP-13 \\
\hline U2-3 & V & A & A & A & A & A & A & A & A & A & A & A & A & A & A & A & A & VP-14 \\
\hline U2-4 & A & A & A & A & A & A & A & A & A & A & A & A & V & A & A & V & A & VP-15 \\
\hline U2-5 & A & $\mathrm{A}$ & A & A & A & V & A & A & $\mathrm{V}$ & A & A & A & A & A & A & A & A & VP-16 \\
\hline U2-6 & $\mathrm{V}$ & A & A & A & A & A & A & A & A & A & A & A & A & A & A & $\mathrm{V}$ & $\mathrm{V}$ & VP-17 \\
\hline U2-7 & A & A & A & A & A & A & A & A & V & A & A & A & A & A & A & $\mathrm{V}$ & A & VP-18 \\
\hline $\mathrm{U} 2-8$ & A & $\mathrm{A}$ & A & A & A & V & A & A & V & V & A & A & A & A & A & V & A & VP-19 \\
\hline U2-9 & A & A & A & A & A & A & A & A & A & A & A & $\mathrm{V}$ & A & A & A & $\mathrm{V}$ & $\mathrm{V}$ & VP-20 \\
\hline $\mathrm{U} 2-10$ & A & A & A & A & A & A & A & A & A & A & A & A & A & A & A & A & A & VP-7 \\
\hline U2-11 & V & $\mathrm{A}$ & V & A & V & A & A & A & A & A & A & A & A & A & A & A & A & VP-21 \\
\hline $\mathrm{U} 2-13$ & V & A & V & A & A & $\mathrm{V}$ & A & A & V & A & A & A & V & A & A & $\mathrm{V}$ & A & VP-22 \\
\hline $\mathrm{U} 2-14$ & $\mathrm{~V}$ & A & V & A & A & $\mathrm{V}$ & A & A & V & A & A & $\mathrm{V}$ & $\mathrm{V}$ & A & A & $\mathrm{V}$ & $\mathrm{V}$ & VP-23 \\
\hline U3-1 & V & A & V & A & A & V & A & A & V & A & A & V & V & A & A & V & A & VP-24 \\
\hline U3-2 & V & A & V & A & A & V & A & A & V & A & A & $\mathrm{V}$ & $\mathrm{V}$ & A & A & $\mathrm{V}$ & $\mathrm{V}$ & VP-23 \\
\hline U3-3 & V & A & V & A & A & $\mathrm{V}$ & A & A & $\mathrm{V}$ & A & A & $\mathrm{V}$ & $\mathrm{V}$ & A & A & $\mathrm{V}$ & $\mathrm{V}$ & VP-23 \\
\hline U3-4 & V & A & V & A & A & V & A & A & V & A & A & V & $\mathrm{V}$ & A & A & $\mathrm{V}$ & $\mathrm{V}$ & VP-23 \\
\hline U3-5 & A & A & V & A & A & $\mathrm{V}$ & A & A & V & A & $\mathrm{V}$ & $\mathrm{V}$ & $\mathrm{V}$ & A & A & $\mathrm{V}$ & $\mathrm{V}$ & VP-25 \\
\hline U4-6 & V & A & A & A & $\mathrm{V}$ & A & A & A & A & A & A & A & A & A & $\mathrm{V}$ & V & A & VP-26 \\
\hline U4-8 & V & A & A & V & V & A & A & A & A & A & A & V & A & A & V & V & A & VP-27 \\
\hline U4-10 & V & A & V & V & $\mathrm{V}$ & A & A & A & A & A & A & V & A & A & A & $\mathrm{V}$ & A & VP-28 \\
\hline U5-1 & A & A & A & A & A & A & A & A & A & A & A & A & A & A & A & A & A & VP-7 \\
\hline U5-2 & A & A & V & V & A & A & A & A & $\mathrm{V}$ & A & A & $\mathrm{V}$ & A & A & $\mathrm{V}$ & A & A & VP-29 \\
\hline U5-3 & A & A & A & A & $\mathrm{V}$ & A & A & A & A & A & A & V & A & A & A & $\mathrm{V}$ & A & VP-2 \\
\hline U5-4 & A & A & V & V & $\mathrm{V}$ & A & A & A & $\mathrm{V}$ & A & A & V & A & A & $\mathrm{V}$ & V & $\mathrm{V}$ & VP-30 \\
\hline U5-6 & A & A & A & A & A & A & A & A & A & A & A & A & A & A & $\mathrm{V}$ & $\mathrm{V}$ & A & VP-31 \\
\hline U5-7 & A & A & A & A & A & A & A & A & A & A & A & A & A & A & V & A & $\mathrm{V}$ & VP-32 \\
\hline U5-8 & V & A & A & A & $\mathrm{V}$ & A & A & A & A & A & A & V & A & A & A & V & A & VP-33 \\
\hline U5-9 & A & A & V & V & A & A & A & A & V & A & A & V & A & A & V & $\mathrm{V}$ & $\mathrm{V}$ & VP-34 \\
\hline U5-11 & A & A & A & V & $\mathrm{V}$ & A & A & A & A & A & A & A & A & A & V & A & A & VP-35 \\
\hline U6-1 & A & $\mathrm{A}$ & A & A & A & A & A & A & A & A & $\mathrm{A}$ & A & A & A & A & A & A & VP-7 \\
\hline U6-2 & V & A & V & A & A & V & A & A & V & A & A & V & V & A & A & V & V & VP-23 \\
\hline U6-3 & V & A & V & A & A & A & A & A & A & A & A & A & A & A & A & $\mathrm{V}$ & $\mathrm{V}$ & VP-36 \\
\hline U6-7 & V & A & V & A & A & V & A & A & V & A & V & V & A & A & A & V & V & VP-37 \\
\hline U6-8 & V & A & V & A & A & V & A & A & A & A & A & V & A & A & A & A & V & VP-38 \\
\hline U7-1 & V & A & V & V & V & A & A & A & A & A & A & A & A & A & V & V & A & VP-39 \\
\hline U7-3 & V & A & V & V & $\mathrm{V}$ & A & A & A & A & A & A & V & A & A & V & $\mathrm{V}$ & A & VP-40 \\
\hline U7-4 & V & A & V & V & V & A & A & A & A & A & A & A & A & A & $\mathrm{V}$ & $\mathrm{V}$ & A & VP-39 \\
\hline U7-6 & V & A & V & A & V & A & A & A & A & A & A & V & A & A & V & V & A & VP-41 \\
\hline U7-7 & V & A & A & V & A & A & A & A & A & A & A & V & A & A & $\mathrm{V}$ & $\mathrm{V}$ & A & VP-42 \\
\hline U8-1 & A & A & V & V & $\mathrm{V}$ & A & A & A & A & A & A & V & A & A & V & V & A & VP-6 \\
\hline U8-6 & A & A & V & V & V & A & A & A & A & A & A & A & A & A & $\mathrm{V}$ & $\mathrm{V}$ & A & VP-13 \\
\hline U8-8 & A & A & V & A & V & A & A & A & V & A & A & V & A & A & V & V & A & VP-43 \\
\hline U8-9 & A & $\mathrm{A}$ & A & V & $\mathrm{V}$ & A & A & A & A & A & A & $\mathrm{V}$ & A & A & $\mathrm{V}$ & V & A & VP-44 \\
\hline U8-10 & $\mathrm{V}$ & A & V & A & $\mathrm{V}$ & A & A & A & A & A & A & V & A & A & $\mathrm{V}$ & $\mathrm{V}$ & A & VP-41 \\
\hline
\end{tabular}

a Wheat $Y r$ single-gene lines: Yr2, Kalyansona; Yr5, AvSYr5NIL; Yr6, AvSYr6NIL; Yr7, AvSYr7NIL; Yr8, AvSYr8NIL; Yr9, AvSYr9NIL; Yr10, AvSYr10NIL; Yr15, AvSYr15NIL; Yr17, AvSYr17NIL, Yr24, AvSYr24NIL; Yr26, AvSYr26NIL; Yr27, AvSYr27NIL; Yr44, AvSYr44NIL; YrSP,

AvSYrSPNIL; YrTr1, AvSYrTr1NIL; YrExp2, AvS/Exp 1/1-1 Line 74; Yr25, Hugenoot; Yr28, AvSYr28NIL (Sharma-Poudyal et al. 2013; Wan and Chen 2014). $\mathrm{A}=$ avirulent (infection types 0 to 6 ) and $\mathrm{V}=$ virulent (infection types 7 to 9 ).

b Isolates U1-1 to U16-5 are single-uredinium isolates derived from 16 single-aecium samples obtained from naturally infected barberry plants.

c Virulence pattern. 
Avirulence (A) or virulence (V) on $Y r$ single-gene lines ${ }^{\mathrm{a}}$

\begin{tabular}{|c|c|c|c|c|c|c|c|c|c|c|c|c|c|c|c|c|c|c|}
\hline Isolate $^{b}$ & $\overline{Y r 2}$ & Yr5 & Yr6 & $Y r 7$ & Yr8 & Yr9 & Yr10 & Yr15 & Yr17 & Yr24 & Yr27 & Yr44 & YrSP & YrTr1 & YrExp2 & Yr25 & Yr28 & $V^{c}$ \\
\hline U9-1 & $\mathrm{A}$ & A & $\mathrm{V}$ & A & $\mathrm{A}$ & $\mathrm{V}$ & A & A & A & $\mathrm{A}$ & $\mathrm{A}$ & $\mathrm{V}$ & A & A & A & A & $\mathrm{V}$ & VP-45 \\
\hline U9-2 & A & A & V & A & A & A & A & A & A & A & A & V & A & A & A & $\mathrm{V}$ & A & VP-46 \\
\hline U9-3 & A & A & A & A & A & A & A & A & A & A & A & A & A & A & A & A & A & VP-7 \\
\hline U9-4 & A & A & V & A & A & $\mathrm{V}$ & A & A & A & A & A & V & $\mathrm{V}$ & A & A & $\mathrm{V}$ & V & VP-47 \\
\hline U9-5 & A & A & V & A & A & $\mathrm{V}$ & A & A & $\mathrm{V}$ & A & A & A & $\mathrm{V}$ & A & A & A & V & VP-48 \\
\hline U9-6 & V & A & A & A & A & A & A & A & $\mathrm{V}$ & A & A & V & $\mathrm{V}$ & A & A & $\mathrm{V}$ & V & VP-49 \\
\hline U9-7 & A & A & V & A & A & V & A & A & A & A & $\mathrm{V}$ & $\mathrm{A}$ & $\mathrm{V}$ & A & A & $\mathrm{V}$ & A & VP-50 \\
\hline U9-8 & A & A & V & A & A & A & A & A & A & A & A & A & A & A & A & A & $\mathrm{V}$ & VP-51 \\
\hline U9-9 & A & A & V & A & A & $\mathrm{V}$ & A & A & A & A & A & $\mathrm{V}$ & A & A & A & $\mathrm{V}$ & $\mathrm{V}$ & VP-52 \\
\hline U10-6 & A & A & A & A & A & A & A & A & A & A & A & A & A & A & A & A & A & VP-7 \\
\hline U10-8 & A & A & V & A & A & $\mathrm{V}$ & A & A & V & A & A & V & $\mathrm{V}$ & A & A & A & A & VP-53 \\
\hline U11-1 & A & A & A & V & $\mathrm{V}$ & A & A & A & A & A & A & V & A & A & A & $\mathrm{V}$ & A & VP-54 \\
\hline U11-2 & A & A & A & A & A & V & A & A & V & A & A & V & A & A & $\mathrm{V}$ & $\mathrm{V}$ & A & VP-55 \\
\hline U11-3 & A & A & V & A & A & A & A & A & $\mathrm{V}$ & V & A & A & A & A & $\mathrm{V}$ & $\mathrm{V}$ & A & VP-56 \\
\hline U11-4 & A & A & V & A & V & A & A & A & A & A & A & A & A & A & $\mathrm{V}$ & $\mathrm{V}$ & V & VP-57 \\
\hline U11-5 & A & A & $\mathrm{V}$ & A & A & A & A & A & A & A & A & $\mathrm{V}$ & A & A & A & $\mathrm{V}$ & A & VP-46 \\
\hline U11-6 & A & A & $\mathrm{V}$ & $\mathrm{V}$ & $\mathrm{V}$ & A & A & A & A & A & A & V & A & A & $\mathrm{V}$ & A & A & VP-58 \\
\hline U11-7 & A & A & V & V & A & $\mathrm{V}$ & A & A & $\mathrm{V}$ & A & A & $\mathrm{V}$ & A & A & $\mathrm{V}$ & $\mathrm{V}$ & $\mathrm{V}$ & VP-59 \\
\hline U11-9 & A & A & $\mathrm{V}$ & A & $\mathrm{V}$ & A & A & A & A & A & A & $\mathrm{V}$ & A & A & $\mathrm{V}$ & $\mathrm{V}$ & A & VP-60 \\
\hline U11-10 & A & A & A & V & $\mathrm{V}$ & A & A & A & $\mathrm{V}$ & A & A & V & A & A & $\mathrm{V}$ & $\mathrm{V}$ & A & VP-61 \\
\hline U11-11 & V & A & V & $\mathrm{V}$ & $\mathrm{V}$ & A & A & A & A & A & A & $\mathrm{V}$ & A & $\mathrm{V}$ & $\mathrm{V}$ & $\mathrm{V}$ & A & VP-62 \\
\hline U12-1 & A & A & $\mathrm{V}$ & $\mathrm{V}$ & A & $\mathrm{V}$ & A & A & $\mathrm{V}$ & A & A & $\mathrm{V}$ & $\mathrm{V}$ & A & $\mathrm{V}$ & $\mathrm{V}$ & A & VP-63 \\
\hline U12-3 & A & A & V & V & A & A & A & A & A & A & A & V & A & A & $\mathrm{V}$ & $\mathrm{V}$ & A & VP-64 \\
\hline U12-4 & A & A & A & V & $\mathrm{V}$ & A & A & A & A & A & A & A & A & A & $\mathrm{V}$ & $\mathrm{V}$ & A & VP-65 \\
\hline U12-5 & A & A & $\mathrm{V}$ & A & $\mathrm{V}$ & A & A & A & A & A & A & $\mathrm{V}$ & A & A & A & $\mathrm{V}$ & A & VP-66 \\
\hline U12-7 & A & A & $\mathrm{V}$ & V & V & A & A & A & A & A & A & A & A & A & A & $\mathrm{V}$ & A & VP-67 \\
\hline U12-10 & A & A & A & A & A & A & A & A & A & A & A & A & A & A & $\mathrm{V}$ & A & A & VP-68 \\
\hline U13-1 & A & A & V & V & V & A & A & A & A & A & A & A & A & A & $\mathrm{V}$ & $\mathrm{V}$ & A & VP-13 \\
\hline U13-2 & A & A & A & A & $\mathrm{V}$ & A & A & A & $\mathrm{V}$ & A & A & V & A & A & $\mathrm{V}$ & $\mathrm{V}$ & A & VP-69 \\
\hline U13-3 & A & A & A & A & A & A & A & A & A & A & A & A & A & A & A & $\mathrm{V}$ & A & VP-70 \\
\hline U13-4 & A & A & A & A & A & A & A & A & A & A & A & A & A & A & A & $\mathrm{V}$ & A & VP-70 \\
\hline U13-5 & $\mathrm{A}$ & A & A & V & $\mathrm{V}$ & A & A & A & A & A & A & A & $\mathrm{A}$ & A & $\mathrm{V}$ & $\mathrm{V}$ & A & VP-65 \\
\hline U13-6 & A & A & A & $\mathrm{V}$ & $\mathrm{V}$ & A & A & A & $\mathrm{V}$ & A & A & $\mathrm{V}$ & A & A & $\mathrm{V}$ & $\mathrm{V}$ & A & VP-61 \\
\hline U13-7 & A & A & V & $\mathrm{V}$ & $\mathrm{V}$ & A & A & A & $\mathrm{V}$ & A & A & A & A & A & A & A & A & VP-71 \\
\hline U13-8 & $\mathrm{V}$ & A & V & $\mathrm{V}$ & A & A & A & A & V & A & A & V & A & A & $\mathrm{V}$ & $\mathrm{V}$ & A & VP-72 \\
\hline U13-9 & V & A & $\mathrm{V}$ & A & $\mathrm{V}$ & $\mathrm{V}$ & A & A & $\mathrm{V}$ & A & A & $\mathrm{V}$ & $\mathrm{V}$ & A & A & $\mathrm{V}$ & $\mathrm{V}$ & VP-73 \\
\hline U13-10 & A & A & A & $\mathrm{V}$ & $\mathrm{V}$ & A & A & A & A & A & A & A & A & A & $\mathrm{V}$ & $\mathrm{V}$ & A & VP-65 \\
\hline U13-11 & A & A & A & V & A & V & A & A & A & A & A & V & A & A & $\mathrm{V}$ & $\mathrm{V}$ & A & VP-74 \\
\hline U14-1 & A & A & $\mathrm{V}$ & A & $\mathrm{V}$ & A & A & A & A & A & A & A & A & A & $\mathrm{V}$ & $\mathrm{V}$ & A & VP-75 \\
\hline U14-2 & A & A & $\mathrm{V}$ & A & $\mathrm{V}$ & A & A & A & $\mathrm{V}$ & A & A & $\mathrm{V}$ & A & A & A & $\mathrm{V}$ & A & VP-76 \\
\hline U14-3 & $\mathrm{V}$ & A & $\mathrm{V}$ & $\mathrm{V}$ & $\mathrm{V}$ & A & A & A & $\mathrm{V}$ & A & A & $\mathrm{V}$ & A & A & $\mathrm{V}$ & $\mathrm{V}$ & A & VP-77 \\
\hline U14-5 & A & A & A & A & A & A & A & A & A & A & A & A & A & A & A & $\mathrm{V}$ & A & VP-70 \\
\hline U14-7 & A & A & A & A & A & A & A & A & A & A & A & V & A & A & $\mathrm{V}$ & A & A & VP-78 \\
\hline U14-8 & A & A & V & V & $\mathrm{V}$ & A & A & A & V & A & A & A & $\mathrm{A}$ & A & A & $\mathrm{V}$ & A & VP-79 \\
\hline U15-1 & A & A & $\mathrm{V}$ & A & $\mathrm{V}$ & A & A & A & $\mathrm{V}$ & $\mathrm{V}$ & A & $\mathrm{V}$ & A & A & $\mathrm{V}$ & $\mathrm{V}$ & A & VP-80 \\
\hline U15-2 & $\mathrm{V}$ & A & V & A & V & A & A & A & $\mathrm{V}$ & A & A & A & A & A & $\mathrm{V}$ & A & A & VP-81 \\
\hline U15-3 & A & A & A & $\mathrm{V}$ & V & A & A & A & A & A & A & A & A & A & $\mathrm{V}$ & $\mathrm{V}$ & A & VP-65 \\
\hline U15-4 & A & A & $\mathrm{V}$ & A & A & A & A & A & A & A & A & A & A & A & $\mathrm{V}$ & A & A & VP-82 \\
\hline U15-5 & A & A & A & A & $\mathrm{V}$ & A & A & A & $\mathrm{V}$ & A & A & $\mathrm{V}$ & A & A & $\mathrm{V}$ & $\mathrm{V}$ & A & VP-69 \\
\hline U15-6 & A & A & A & V & V & A & A & A & A & A & A & V & A & A & $\mathrm{V}$ & $\mathrm{V}$ & A & VP-44 \\
\hline U15-7 & A & A & A & A & $\mathrm{V}$ & A & A & A & A & A & A & $\mathrm{V}$ & A & A & A & $\mathrm{V}$ & A & VP-2 \\
\hline U15-8 & A & A & A & A & $\mathrm{V}$ & A & A & A & $\mathrm{V}$ & A & A & $\mathrm{V}$ & A & A & A & $\mathrm{V}$ & A & VP-83 \\
\hline U15-9 & A & A & A & A & V & A & A & A & A & A & A & V & A & A & A & $\mathrm{V}$ & A & VP-2 \\
\hline U15-10 & A & A & $\mathrm{V}$ & A & A & A & A & A & $\mathrm{V}$ & A & A & A & A & A & $\mathrm{V}$ & $\mathrm{V}$ & A & VP-84 \\
\hline U15-11 & A & A & A & A & A & V & A & A & V & A & A & V & A & A & A & $\mathrm{V}$ & A & VP-85 \\
\hline U16-1 & $\mathrm{V}$ & A & V & A & A & V & A & A & A & $\mathrm{A}$ & A & V & A & A & A & $\mathrm{V}$ & A & VP-86 \\
\hline U16-2 & V & A & V & V & V & A & A & A & $\mathrm{V}$ & A & A & $\mathrm{V}$ & A & A & $\mathrm{V}$ & $\mathrm{V}$ & A & VP-77 \\
\hline U16-4 & $\mathrm{V}$ & A & V & V & A & A & A & A & A & A & A & V & A & A & A & $\mathrm{V}$ & A & VP-87 \\
\hline U16-5 & A & A & A & V & A & A & A & A & A & A & A & V & A & A & A & $\mathrm{V}$ & A & VP-88 \\
\hline $\mathrm{V}(\%)$ & 28.0 & 0.0 & 52.5 & 33.1 & 48.3 & 20.3 & 0.0 & 0.0 & 34.8 & 2.5 & 2.5 & 55.1 & 13.6 & 0.9 & 48.3 & 72.9 & 21.2 & \\
\hline
\end{tabular}

0-to-4 scale for P. graminis f. sp. tritici) or no symptom (IT 0) (Table 2). The species of these aecia could not be determined in the present study. Simultaneously, 14 P. graminis f. sp.tritici samples were obtained, also at a low frequency $(0.13 \%)$, from the tested SA samples.
Virulence patterns of the $P$. striiformis f. sp. tritici SA samples. The 16 P. striiformis $\mathrm{f}$. sp. tritici SA samples had 15 different virulence patterns on the Chinese differential genotypes (Table 3). Compared with the 67 P. striiformis f. sp. tritici races from wheat as reported by Zhan et al. (2012), 14 of the patterns were different 
from those of the previously reported races. The two isolates (U2 and U3) collected from B. aggregata in Gansu had a virulence pattern similar to CYR32, a predominant $P$. striiformis f. sp. tritici race in the region. In addition, SA isolate $\mathrm{U} 10$ from $B$. shensiana in Shaanxi had a virulence pattern similar to that of CYR32, with the only difference in its avirulence on wheat differential Danish 1. Isolates U5 and U7, also from B. shensiana in Shaanxi, had virulence patterns similar to CYR20, with additional virulences on differentials Virgilio and Suwon 11 in both U5 and U7 and additional virulence on differential Jubilejina 2 in U5. Isolate U4 from B. polyantha in Tibet had the same virulence pattern as CYR20, except for its virulence on differentials Fengchan 3 and Suwon 11. None of the SA samples were virulent to Zhong 4 ( $Y r$ gene unknown), Triticum spelta ablum (Yr5), and Guinong 22 (Yr26), similar to the $P$. striiformis f. sp. tritici populations from wheat crops.

The 16 P. striiformis f. sp. tritici SA samples were also tested on the $17 \mathrm{Yr}$ single-gene lines (Table 4). Isolates U2 and U3 produced the same virulence patterns as race CYR32 on the $\mathrm{Yr}$ single-gene lines (data not shown). None of the 16 SA samples produced identical virulence patterns, similar to the results obtained with the Chinese differentials.

Most of the SA samples had mixed IT on some of the differential genotypes and all of the SA samples had mixed IT on many $Y r$ singlegene lines, indicating that the SA samples comprised isolates with different virulence patterns. Therefore, $\mathrm{SU}$ isolates were established from each of the SA samples and tested on the $17 \mathrm{Yr}$ single-gene lines.

Virulence patterns of SU isolates. From the $16 \mathrm{SA}$ samples, 118 $\mathrm{SU}$ isolates were obtained. The SU isolates were identified to have 88 virulence patterns based on the IT data of the $17 \mathrm{Yr}$ single-gene lines (Table 5). The high pattern/isolate ratio (1: 1.3 ) clearly show that these isolates were produced through sexual reproduction.

The 88 virulence patterns had relatively narrow virulence spectra, ranging from 0 to 9 with a mean of 4 virulences (Table 5) compared with their SA samples (Table 4). The frequencies of the virulences to the $17 \mathrm{Yr}$ genes among the $118 \mathrm{SU}$ isolates are shown at the bottom of Table 5. No virulences were detected for $Y r 5, Y r 10$, and $Y r 15$; virulences to $Y r T r 1, Y r 24$, and $Y r 27$ were extremely low ( $<3 \%)$; those to $\operatorname{YrSP}, \operatorname{Yr} 9, \mathrm{Yr} 28$, and $Y r 2$ were low (13.6 to 28.0\%); those to $\operatorname{Yr} 7$, Yr17, $Y r 8$, and $Y r E x p 2$ were moderate (33.1 to $48.3 \%$ ); and those to $Y r 6, Y r 44$, and $Y r 25$ were high (52.5 to $72.9 \%$ ).

Most of the virulence patterns were represented by only one isolate, while only $15(17 \%)$ patterns had two or more isolates (Table 5). Nine virulence patterns (VP-1, VP-6, VP-39, VP-41, VP-44, VP-46, VP-61, VP-69, and VP-77) each had two isolates, two patterns (VP-13 and VP-70) each had three isolates, two patterns (VP-2 and VP-65) each had four isolates, one pattern (VP-23) had six isolates, and one pattern (VP-7) had eight isolates. Isolates with an identical virulence pattern were generally from different SA samples. Some of the pattern-shared isolates were from different Berberis spp. and different provinces. For example, VP-7 consisted of five SU isolates (U1-7, U1-10, U2-1, U2-10, and U6-1) from B. aggregata in Gansu and three isolates (U5-1, U9-3, and U10-6) from B. shensiana in Shaanxi.

\section{Discussion}

In this study, 9,297 aecia were individually tested on Mingxian 169 wheat, from which 16 P. striiformis f. sp. tritici SA samples were obtained. These isolates and their derived $118 \mathrm{SU}$ isolates were clearly shown by virulence tests to be produced from sexual reproduction on barberry plants. This study, together with a previous study (Zhao et al. 2013), provided direct evidence that $P$. striiformis $\mathrm{f}$. sp. tritici sexual reproduction exists in China, as was previously speculated based on marker analysis of the $P$. striiformis f. sp. tritici population from wheat fields (Mboup et al. 2009). Compared with the previous study reporting only four isolates from Gansu and Shaanxi (Zhao et al. 2013), the present study has extended the geographic areas where $P$. striiformis $\mathrm{f}$. sp. tritici sexual reproduction occurs from the northwest to southwest of China.
Many regions in western China, especially southern Gansu and northwestern Sichuan, are regarded as hotspots for wheat stripe rust in China according to the history of the disease epidemics (Li and Zeng 2002). In these regions, $P$. striiformis f. sp. tritici oversummers or overwinters in the uredinial stage and provides inoculum to initiate infection on wheat within and among the regions and further spread to regions in eastern China (Chen et al. 2009; Shan et al. 1998). More importantly, most of the $P$. striiformis $\mathrm{f}$. sp. tritici races (approximately $90 \%$ ), particularly those that have overcome resistance in major wheat cultivars, were identified first in the hotspot regions (Chen et al. 2009; Huang et al. 2014; Kang and Li 1984; Kang et al. 1987, 1994; Liu et al. 2010; Wang et al. 1986). Based on our previous surveys, there are abundant barberry plants with rust fungal aecia adjacent to wheat fields in Gansu and Shaanxi provinces but only four $P$. striiformis f. sp. tritici samples were isolated (Zhao et al. 2013). The present study expanded our knowledge of rusting barberry plants existing in Tibet and Xinjiang. Interestingly, we isolated $P$. striiformis $\mathrm{f}$. sp. tritici from the aecial samples collected from Tibet but not from those of Xinjiang. Thus, the barberry bushes adjacent to wheat fields may provide great opportunities for the spread of basidiospores from wheat straws to infect barberry and aeciospores from barberry to infect wheat. However, whether barberry plants provide aeciospores to initiate stripe rust in wheat fields needs further study because stripe rust was generally observed in wheat fields when we saw aecia on barberry plants.

In the present study, only 16 of 9,297 aecia were clearly identified as $P$. striiformis f. sp.tritici, indicating a very low frequency $(0.17 \%)$. Stripe rust is the most frequent disease of wheat in the surveyed regions. The low frequency of $P$. striiformis $\mathrm{f}$. sp. tritici on barberry plants may suggest really low infection by the pathogen under natural conditions. The direct connection between the stem rust fungi on alternate hosts and cereal crops have been well known for centuries (Roelfs 1982; Zadoks and Bouwman 1985). However, no such phenomenon has been reported for stripe rust. The low infection frequency may explain why apparent connections between stripe rust of wheat and barberry have not been observed. The low level of $P$. striiformis f. sp. tritici infection on barberry could be due to a low survival rate of teliospores after winter. Further studies are needed to test this hypothesis.

More than $99 \%$ of the individual aecia were not identified as either $P$. striiformis f. sp. tritici or $P$. graminis f. sp. tritici because they did not produce uredinia on the inoculated wheat plants. In addition to $P$. striiformis and $P$. graminis, many other Puccinia spp. are able to infect barberry plants. These species include $P$. brachypodii (Payak 1965); P. poa-nemoralis, P. pygmaea, P. montanensis, and P. brachypodii-phoenicoidis (Cummins and Greene 1966); P. arrhenatheri (Naef et al. 2002); and P. striiformis f. sp. poae (Jin et al. 2010). Therefore, most aecial pustules observed on barberry plants might be other Puccinia spp. or formae speciales of $P$. striiformis and $P$. graminis. Further studies are needed to identify rust fungal species and their frequencies on barberry plants. We are currently developing molecular markers and using sequencing techniques to study the different Puccinia spp. on barberry. In addition, some of the aecial inoculations might not have been successful, due to possible reduction of the viability of aecia during the travel and storage before inoculation. Some aecia might be $P$. striiformis f. sp. tritici but avirulent on Mingxian 169. To further improve the inoculation and identification efficiencies, either wheat genotypes universally susceptible to $P$. striiformis $\mathrm{f}$. sp. tritici and $P$. graminis f. sp. tritici or different genotypes susceptible to different rust fungi should be used in tests with split aecial samples. Together with suitable molecular markers and sequencing, accurate frequencies of various Puccinia spp. on barberry should be determined.

Two of the SA samples had an overall virulence pattern similar to the predominant $P$. striiformis $\mathrm{f}$. sp. tritici race CYR32 in the region (Chen et al. 2009; Wan et al. 2004; Zhan et al. 2012). This result more likely indicated that the sources of inoculum (teliospores on wheat straw) for infection of the barberry plants were from the $P$. striiformis f. sp. tritici population in the nearby wheat fields. Most virulence patterns identified from the $118 \mathrm{SU}$ isolates have not been found in wheat isolates. This could be due to stripe rust resistance conferred 
by various resistance genes in wheat cultivars. Because most isolates had relatively narrow virulence spectra, they were unable to infect commercially grown wheat cultivars. For example, a large number of wheat cultivars have resistance gene $\operatorname{Yr} 9$ (Zeng et al. 2014), and this gene alone can prevent infection by $80 \%$ of the SU isolates. Yr24 (= Yr26) is in many of the wheat cultivars developed in the recent years (Zeng et al. 2014), and cultivars with that gene can prevent infection of $97.5 \%$ of the SU isolates detected in the present study. The present study shows that $\operatorname{Yr} 5, \operatorname{Yr} 10$, and $\operatorname{Yr} 15$ are still resistant against all virulent patterns identified from the sexual population, as well as against the predominant races such as CYR32 in the asexual population. However, further studies should be conducted to determine virulence exchanges between the sexual population and asexual population.

Although $P$. striiformis $\mathrm{f}$. sp. tritici on barberry plants may not play a major role in initiating stripe rust on wheat crops, it may generate new and unique races which could damage wheat crops, even at a low frequency. To reduce the potential of new races damaging wheat crops, race-nonspecific resistance or diverse resistance genes should be used to develop wheat cultivars with stripe rust resistance, no matter whether or not $P$. striiformis $\mathrm{f}$. sp. tritici infecting barberry plants can provide initial inoculum to infect wheat crops or generate new races attacking specific wheat cultivars.

\section{Acknowledgments}

This study was supported by National Key Basic Research Program of China (2013CB127700), International Science \& Technology Cooperation Program of China (2011DFG32990), the earmarked fund for Modern Agro-industry Technology Research System Project in China (CARS-3-1-11), National Natural Science Foundation of China (31271986), the 111 Project from the Ministry of Education of China (B07049), and Program for New Century Excellent Talents in University.

\section{Literature Cited}

Ali, S., Gladieux, P., Rahman, H., Saqib, M. S., Fiaz, M., Ahmad, H., Leconte, M., Gautier, A., Justesen, A. F., Hovmøller, M. S., Enjalbert, J., and de VallavieillePope, C. 2014. Inferring the contribution of sexual reproduction, migration and off-season survival to the temporal maintenance of microbial populations: A case study on the wheat fungal pathogen Puccinia striiformis f. sp. tritici. Mol. Ecol. 23:603-617.

Chen, W. Q., Wellings, C., Chen, X. M., Kang, Z. S., and Liu, T. G. 2014. Wheat stripe (yellow) rust caused by Puccinia striiformis f. sp. tritici. Mol. Plant Pathol. 15:433-446.

Chen, W. Q., Wu, L. R., Xu, S. C., Jin, S. L., Peng, Y. L., and Wang, B. T. 2009. Race dynamics, diversity, and virulence evolution in Puccinia striiformis f. sp. tritici, the causal agent of wheat stripe rust in China from 2003 to 2007. Plant Dis. 93:1093-1101.

Chen, X. M. 2005. Epidemiology and control of stripe rust on wheat. Can. J. Plant Pathol. 27:314-337.

Chen, X. M., Penman, L., Wan, A. M., and Cheng, P. 2010. Virulence races of Puccinia striiformis f. sp. tritici in 2006 and 2007 and development of wheat stripe rust and distributions, dynamics, and evolutionary relationships of races from 2000 to 2007 in the United States. Can. J. Plant Pathol. 32:315-333.

Cummins, G. B., and Greene, H. C. 1966. A review of the grass rust fungi that have uredial paraphyses and aecia on Berberis-Mahonia. Mycologia 58:702-721.

Duan, X. Y., Tellier, A., Wan, A. M., Leconte, M., de Vallavieille-Pope, C., and Enjalbert, J. 2010. Puccinia striiformis f. sp. tritici presents high diversity and recombination in the over-summering zone of Gansu, China. Mycologia 102:44-53.

Harder, D. E. 1984. Developmental ultrastructure of hyphae and spores. Pages 333-373 in: The Cereal Rusts. Bushnell W. R., and Roelfs, A. P., eds. Academic Press, New York.

Hu, X. P., Li, J. J., Wang, Y. T., Wang, B. T., Li, Q., Kang, Z. S., Yang, M. N., Peng, Y. L., Liu, T. G., Chen, W. Q., and Xu, X. M. 2012. Race composition of Puccinia striiformis f. sp. tritici in Tibet, China. Plant Dis. 96:1615-1620.

Huang, J., Jia, Q. Z., Jin, S. L., Cao, S. Q., Zhang, B., Sun, Z. Y., Luo, H. S., and Wang, X. M. 2014. Population changes of Puccinia striiformis $\mathrm{f}$. sp. tritici in Gansu Province during 2010-2012. Plant Prot. 40:101-105.

Jin, Y., Szabo, L. J., and Carson, M. 2010. Century-old mystery of Puccinia striiformis life history solved with the identification of Berberis as an alternate host. Phytopathology 100:432-435.

Kang, Z. S., and Li, Z. Q. 1984. Discovery of a normal T type new pathogenic strain to Lovrin 10. J. Northwest A\&F Univ. 4:18-28.

Kang, Z. S., Li, Z. Q., and Shang, H. S. 1993. A new isolate produced by the heterokaryosis of wheat stripe rust. J. Northwest A\&F Univ. 21:97-99.

Kang, Z. S., Li, Z. Q., Shang, H. S., Lu, H. P., and Liu, X. D. 1994. Discovery of new isolates virulent to wheat cultivars of Mianyang system in wheat stripe rust. J. Northwest A\&F Univ. 22:7-11.
Kang, Z. S., Li, Z. Q., and Zhang, S. C. 1987. A preliminary study of pathogenic strain to Lovrin 13 of yellow rust of wheat. J. Northwest A\&F Univ. 15:105-106.

Kang, Z. S., Zhao, J., Han, D. J., Zhang, H. C., Wang, X. J., Wang, C. F., Han, Q. M., Guo, J., and Huang, L. L. 2010. Status of wheat rust research and control in China. Pages 50-69 in: BGRI 2010 Tech. Workshop, St. Petersburg, Russia.

Li, Z. Q., and Zeng, S. M. 2002. Wheat Stripe Rust in China. China Agriculture Press, Beijing.

Line, R. F., and Qayoum, A. 1992. Virulence, aggressiveness, evolution, and distribution of races of Puccinia striiformis (the cause of stripe rust of wheat) in North America, 1968-87. U.S. Dep. Agric. Tech. Bull. 1788.

Little, R., and Manners, J. G. 1969. Somatic recombination in yellow rust of whea (Puccinia striiformis): I. The production and possible origin of two new physiologic races. Trans. Br. Mycol. Soc. 53:251-258.

Liu, T. G., Peng, Y. L., Chen, W. Q., and Zhang, Z. Y. 2010. First detection of virulence in Puccinia striiformis f. sp. tritici in China to resistance genes Yr24 (=Yr26) present in wheat cultivar Chuanmai 42. Plant Dis. 94:1163.

Lu, N. H., Zhan, G. M., Wang, J. F., Huang, L. L., and Kang, Z. S. 2009. Molecular evidence of somatic genetic recombination of Puccinia striiformis $\mathrm{f}$. sp. tritici in China. Acta Phytopathol. Sin. 39:561-568.

Mboup, M., Leconte, M., Gautier, A., Wan, A. M., Chen, W. Q., de VallavieillePope, C., and Enjalbert, J. 2009. Evidence of genetic recombination in whea yellow rust populations of a Chinese oversummering area. Fungal Genet. Biol. 46:299-307.

Milus, E. A., Seyran, E., and McNew, R. 2006. Aggressiveness of Puccinia striiformis f. sp. tritici isolates in the south-central United States. Plant Dis. 90:847-852.

Naef, A., Roy, B. A., Kaiser, R., and Honegger, R. 2002. Insect-mediated reproduction of systemic infections by Puccinia arrhenatheri on Berberis vulgaris. New Phytol. 154:717-730.

Payak, M. M. 1965. Berberis as the aecial host of Puccinia brachypodii in Simla Hills (India). J. Phytopathol. 52:49-54.

Rapilly, F. 1979. Yellow rust epidemiology. Annu. Rev. Phytopathol. 17:59-73.

Rodenhiser, H. A., and Hard-Karrer, A. M. 1947. Evidence of fusion bodies from urediospore germ tubes of cereal rusts on nutrient-solution agar. Phytopathology 37:744-756.

Roelfs, A. P. 1982. Effects of barberry eradication on stem rust in the United States. Plant Dis. 66:177-181.

Shan, W. X., Chen, S. Y., Kang, Z. S., Wu, L. R., and Li, Z. Q. 1998. Genetic diversity in Puccinia striiformis Westend. f. sp. tritici revealed by pathogen genome specific repetitive sequence. Can. J. Bot. 76:587-595.

Sharma-Poudyal, D., Chen, X. M., Wan, A. M., Zhan, G. M., Kang, Z. S., Cao, S. Q., Jin, S. L., Morgounov, A., Akin, B., Mert, Z., Shah, S. J. A., Bux, H., Ashraf, M., Sharma, R. C., Madariaga, R., Puri, K. D., Wellings, C., Xi, K. Q., Wanyera, R., Manninger, K., Ganzález, M. I., Koyda, M., Sanin, S., and Patzek, L. J. 2013. Virulence characterization of international collections of the wheat stripe rust pathogen, Puccinia striiformis f. sp. tritici. Plant Dis. 97:379-386.

Stakman, E. C., Stewart, D. M., and Loegering, W. Q. 1962. Identification of Physiologic Races of Puccinia graminis var. tritici. Page 20 in: United States Department of Agriculture, Agricultural Research Service E617 (revised) Washington D.C.

Stubbs, W. 1985. Stripe rust. Pages 61-101 in: The Cereal Rusts, Vol. II. Diseases, Distribution, Epidemiology, and Control. A. P. Roelfs, and W. R. Bushnell, eds. Academic Press, New York.

Wan, A. M., and Chen, X. M. 2014. Virulence characterization of Puccinia striiformis $\mathrm{f}$. sp. tritici using a new set of $Y r$ single-gene line differentials in the United States in 2010. Plant Dis. 98:1534-1542.

Wan, A. M., Wu, L. R., Jia, Q. Z., and Bi, Y. Q. 2003. Dynamic of physiological race of wheat stripe rust in China from 1997 to 2001. Acta Phytophylac. Sin. 33: 261-266.

Wan, A. M., Zhao, Z. H., Chen, X. M., He, Z. H., Jin, S. L., Jia, Q. Z., Yao, G., Yang, J. X., Wang, B. T., Li, G. B., Bi, Y. Q., and Yuan, Z. Y. 2004. Whea stripe rust epidemic and virulence of Puccinia striiformis f. sp. tritici in China in 2002. Plant Dis. 88:896-904.

Wang, K. N., Hong, X. W., Wu, L. R., Xie, S. X., Meng, Q. Y., and Chen, S. M 1986. The analysis of the resistance of varieties in the wheat stripe rust nurseries in 1951-1983. Acta Phytophylac. Sin. 13:117-124.

Wang, M. N., and Chen, X. M. 2013. First report of Oregon grape (Mahonia aquifolium) as an alternate host for the wheat stripe rust pathogen (Puccinia striiformis f. sp. tritici) under artificial inoculation. Plant Dis. 97:839.

Wellings, C. R. 2011. Global status of stripe rust: A review of historical and curren threats. Euphytica 179:129-141.

Wellings, C. R., and McIntosh, R. A. 1990. Puccinia striiformis f. sp. tritici in Australasia: Pathogenic changes during the first 10 years. Plant Pathol. 39:316-325.

Ying, J. S., and Chen, D. Z. 2001. Lardizabalaceae and Berberidaceae. Pages 50 214 in: Flora of China, Vol. 29. The Editorial Committee of Flora of China, Chinese Academy of Sciences, ed. Science Press, Beijing.

Zadoks, J. C., and Bouwman, J. J. 1985. Epidemiology in Europe. Pages 329-369 in: The Cereal Rusts, Vol II: Diseases, Distribution, Epidemiology, and Control A. P. Roelfs and W. R. Bushnell, eds. Academic Press, New York

Zeng, Q. D., Han, D. J., Wang, Q. L., Yuan, F. P., Wu, J. H., Zhang, L., Wang, X. J., Huang, L. L., Chen, X. M., and Kang, Z. S. 2014. Stripe rust resistance 
and genes in Chinese wheat cultivars and breeding lines. Euphytica 196: 271-284.

Zhan, G. M., Chen, X. M., Kang, Z. S., Huang, L. L., Wang, M. N., Wan, A. M., Cheng, P., Cao, S. Q., and Jin, S. L. 2012. Virulence and molecular comparison of Puccinia striiformis f. sp. tritici populations in China and the United States. Fungal Biol. 116:643-653.

Zhao, J., Wang, L., Wang, Z. Y., Chen, X. M., Zhang, H. C., Yao, J. N., Zhan, G. M., Chen, W., Huang, L. L., and Kang, Z. S. 2013. Identification of eighteen Berberis species as alternate hosts of Puccinia striiformis f. sp. tritici and virulence variation in the pathogen isolates from natural infection of barberry plants in China. Phytopathology 103:927-934.
Zheng, W. M., Chen, S. Y., Kang, Z. S., Wang, Y., Wu, L. R., and Li, Z. Q. 2005. DNA fingerprinting of natural population of Puccinia striiformis f. sp. tritici in Tianshui area. Mycosystema 24:199-206.

Zheng, W. M., Huang, L. L., Huang, J. Q., Wang, X. J., Chen, X. M., Zhao, J., Guo, J., Zhuang, H., Qiu, C. Z., Liu, J., Liu, H. Q., Huang, X. L., Pei, G. L., Zhan, G. M., Tang, C. L., Cheng, Y. L., Liu, M. J., Zhang, J. S., Zhao, Z. T., Zhang, S. J., Han, Q. M., Han, D. J., Zhang, H. C., Zhao, J., Gao, X. N., Wang, J. F., Ni, P. X., Dong, W., Yang, L. F., Yang, H. M., Xu, J. R., Zhang, G. Y., and Kang, Z. S. 2013. High genome heterozygosity and endemic genetic recombination in the wheat stripe rust fungus. Nat. Commun. 4:2673 